\title{
Trans* Community Connection, Health, and Wellbeing: A Systematic Review
}

\author{
Athena D.F. Sherman, PhD, ${ }^{1}$ Kristen D. Clark, MSN, ${ }^{2}$ Kelley Robinson, MSN, ${ }^{1}$ \\ Tara Noorani, MSN, ${ }^{3}$ and Tonia Poteat, $\mathrm{PhD}^{4}$
}

\begin{abstract}
Purpose: Transgender and nonbinary (trans*) people are affected disproportionately by discrimination and violence, contributing to gender minority stress and negative health effects. Transgender community connection (TCC), defined as (i) emotional connectedness (internal sense of belonging) to the trans* community and/or (ii) behavioral participation in the trans* community (observable interaction with other trans* people, in person, online, or through media) may moderate these negative effects on health. This systematic review synthesizes research on links between TCC and health and wellbeing for trans* people.

Methods: Twenty research articles (3 quantitative, 3 mixed methods, and 14 qualitative) linking TCC to health and wellbeing were identified from systematic searches of PubMed, CINAHL, and PsycINFO. Data regarding TCC were extracted, synthesized, and grouped by (i) gender transitioning, (ii) mental health, (iii) sexual health, and (iv) access to care to illustrate the findings.

Results: TCC was linked to several positive outcomes, including improved mental health, increased connection to care, supported exploration of sexual and gender identities, and informed gender transition. For transgender women, especially, TCC was also linked to increased engagement in sex work (as a worker, not a client) and, in some cases, deterred them from getting sexual health testing and treatment due to fear of being observed at specialized clinics and subsequent loss of confidentiality among peers.

Conclusion: These findings highlight the overall protective nature of TCC and a need to consider best practices to ensure confidentiality for community members. The findings can be used to inform the development and adaptation of health care interventions aimed at decreasing the harmful effects of gender minority stress for trans* people.
\end{abstract}

Keywords: behavioral participation, connectedness, critical review, gender minority, LGBT, social support

\section{Introduction}

$\mathbf{T}$ RANSGENDER AND NONBINARY (trans*) people are those whose gender identity is different from their sex assigned at birth. This departure from societal gender norms has been met historically with cisgenderism - a multilevel and systemic prejudicial ideology that delegitimizes or denies a person's ability to self-determine their own gender ${ }^{1,2}$ - and transphobia- "negative emotional or affective responses" toward trans* people. $^{3}$ Cisgenderism and transphobia have been observed as widespread discrimination and violence and have been associated with various socially produced health inequities when comparing outcomes among cisgender and trans* people. ${ }^{4,5}$ Specifically, current research suggests a higher prevalence of sexual assaults against trans* people compared to their cisgender counterparts. ${ }^{6,7}$ This exposure to violence is associated with poor mental and physical health such as a high prevalence of lifetime symptoms of depression $(55 \%),{ }^{8}$ lifetime symptoms of post-traumatic stress $(44 \%),{ }^{9}$ lifetime suicide attempts $(41 \%),{ }^{10}$ and HIV acquisition $(2.8 \%$ incidence). ${ }^{11}$ Health disparities are often compounded by barriers to accessing and engaging in medical care, including discrimination by medical and service providers. ${ }^{12-18}$ Trans* patients report refusal of care by providers, encounter inadequately informed providers, and report postponement of care due to fear of discrimination. ${ }^{12-17}$

The Minority Stress Model presents a framework to help describe the pathways whereby individuals of minority status

\footnotetext{
${ }^{1}$ School of Nursing, Johns Hopkins University, Baltimore, Maryland.

${ }^{2}$ Department of Community Health Systems, School of Nursing, University of California, San Francisco, San Francisco, California.

${ }^{3}$ Nell Hodgson Woodruff School of Nursing, Emory University, Atlanta, Georgia.

${ }^{4}$ School of Medicine, University of North Carolina at Chapel Hill, Chapel Hill, North Carolina.
} 
face external socially produced stressors (e.g., discrimination and violence) and internal stressors (e.g., internalized homophobia-when a sexual minority person directs negative social attitudes and stigma toward themself resulting in negative outcomes) ${ }^{19}$ that contribute to poor mental health. ${ }^{20}$ Within this framework, social support has been identified as a moderating variable, with the potential to decrease the strength of the relationship between stressors and mental health. ${ }^{20}$ The gender minority adaptation of the Minority Stress Model (the Gender Minority Stress Model) highlights trans*-specific stressors such as lack of genderappropriate identity documents, discrimination when seeking medical care, and lack of access to safe public restrooms. ${ }^{21}$ The Gender Minority Stress Model proposes transgender community connection $^{22}$ (TCC) as a moderator capable of reducing the negative effects of gender-related victimization, internalized transphobia, and anticipated future prejudice on mental health among trans* people. ${ }^{22,23}$ TCC has been described in different ways in the literature. ${ }^{21,22}$ For this review, it is defined as (i) emotional connectedness (internal sense of belonging to the trans* community) and/or (ii) behavioral participation in the trans* community (observable interaction with other trans* people, in person, online, or through media). This systematic research review searches qualitative, quantitative, and mixed-methods studies to identify, describe, and synthesize ${ }^{24}$ the possible risks and protective value of TCC and its links to health and wellbeing among trans* people.

\section{Methods}

\section{Search strategy and selection of articles}

In contemporary digital media environments, visibility of and access to affirming trans*-focused media and trans* peers have improved; however, this also comes with additional risk of media-based discrimination and violence. ${ }^{25}$ Contemporary digital media environments have altered the way in which trans* people may choose to interact with community; thus, to focus our findings on research most relevant to the lives and experiences of trans* people today, articles from the preceding decade, published from January 1, 2007 to November 1, 2017, were identified from three databases (PubMed, CINAHL, and PsycINFO) for systematic review. $^{25}$

Search terms were identified in two categories, community connection terms and trans* population terms. Search terms for community connection were as follows: "Community Networks," "community participation," "social participation," “community connect*," "social cohesion," "community cohesion," "social isolation," "collectivism," "isolation," "social support," and "social inclusion." Search terms for trans* population were as follows: "transsexualism," "transgender*," "transsexual*," "gender minority," "FTM," "genderqueer," "crossdresser," “drag king," “drag queen," “non-binary," and "MTF." Additional filters were used to narrow the results by English language only and human subjects only. Inclusion criteria consisted of the following: (i) having transgender participants, (ii) reporting results for trans* individuals separately from those of cisgender individuals, (iii) reporting $\mathrm{QN}$ or QL findings linking TCC with a health outcome, and (iv) being a peer-reviewed primary research article. The follow- ing types of articles were excluded: review articles, psychometric measurement analysis, and dissertations.

Covidence systematic review software ${ }^{26}$ was used to manage article selection. In total, 1166 articles were identified from the initial search, and 942 articles remained after duplicates were removed. Figure 1 shows the Preferred Reporting Items for Systematic Reviews and Meta-Analyses flow diagram. ${ }^{27}$ Title and abstract screening led to the exclusion of 842 articles that did not meet the inclusion criteria. The remaining 100 articles received a full-text review, and 18 met all inclusion criteria. Two additional articles (not indexed in the three reviewed databases) were identified from a hand search of the reference lists of the 18 articles. The final 20 articles were retained for data extraction and qualitative synthesis. ${ }^{22,28-46}$ This systematic review was exempt from institutional review board review at Johns Hopkins University.

\section{Data extraction, analysis, and synthesis}

Data extraction was completed by the first author (A.D.F.S.), and a $25 \%$ audit was performed by an additional team member (T.N.). Extracted data included (i) demographic data on study participants; (ii) forms of TCC measurement; (iii) findings relating TCC to a health outcome; (iv) descriptive findings related to TCC; and (v) study design and methodology. The findings were then compared and grouped according to identified topic and subtopic areas. ${ }^{24}$ The topics were (i) descriptive findings related to TCC and (ii) associations between TCC and health outcomes; the subtopics of area ii were (1) gender transitioning, (2) mental health, (3) sexual health, and (4) access to care.

The first author (A.D.F.S.) and an additional team member (K.R.) completed a critical appraisal of article quality using a modified version of the Checklist for Qualitative Research, and the Checklist for Analytical Cross-Sectional Studies from the Joanna Briggs Institute (JBI; see Table 1). ${ }^{4,48}$ These tools were chosen after a review of multiple quality assessment tools because they provided a multidimensional analysis of quality specific to quantitative and qualitative research. ${ }^{47,48}$ Due to a lack of critical appraisal tools focused on mixed-methods research, articles that employed both quantitative and qualitative methods were evaluated independently on each scale. All discrepancies were discussed and resolved among reviewers. Table 1 contains article quality scores.

\section{Results}

\section{Description of reviewed articles}

The median overall quality of the included articles was 7 out of 10 (interquartile range $[\mathrm{IQR}]=2$ ) for those with mixed methods or qualitative analysis and 5 out of $8(\mathrm{IQR}=5)$ for those with mixed methods or quantitative analysis $(n=17$ mixed methods and qualitative $\mathrm{e}^{28-33,36-46}$ and $n=6$ mixed methods and quantitative $22,34-38$ ). See Table 1 for further details on quality assessment. Only $35 \% \quad(n=7 /$ $20)^{29,30,34,35,38,42,44}$ of articles reported data collection dates, ranging from 1999 to 2011 (all included articles were published no earlier than 2007). Most studies took place in the United States $(n=16 / 20){ }^{22,29-36,38-42,45,46}$ The six international studies reported samples from Canada, ${ }^{22,36,44}$ Croatia, $^{37}$ 


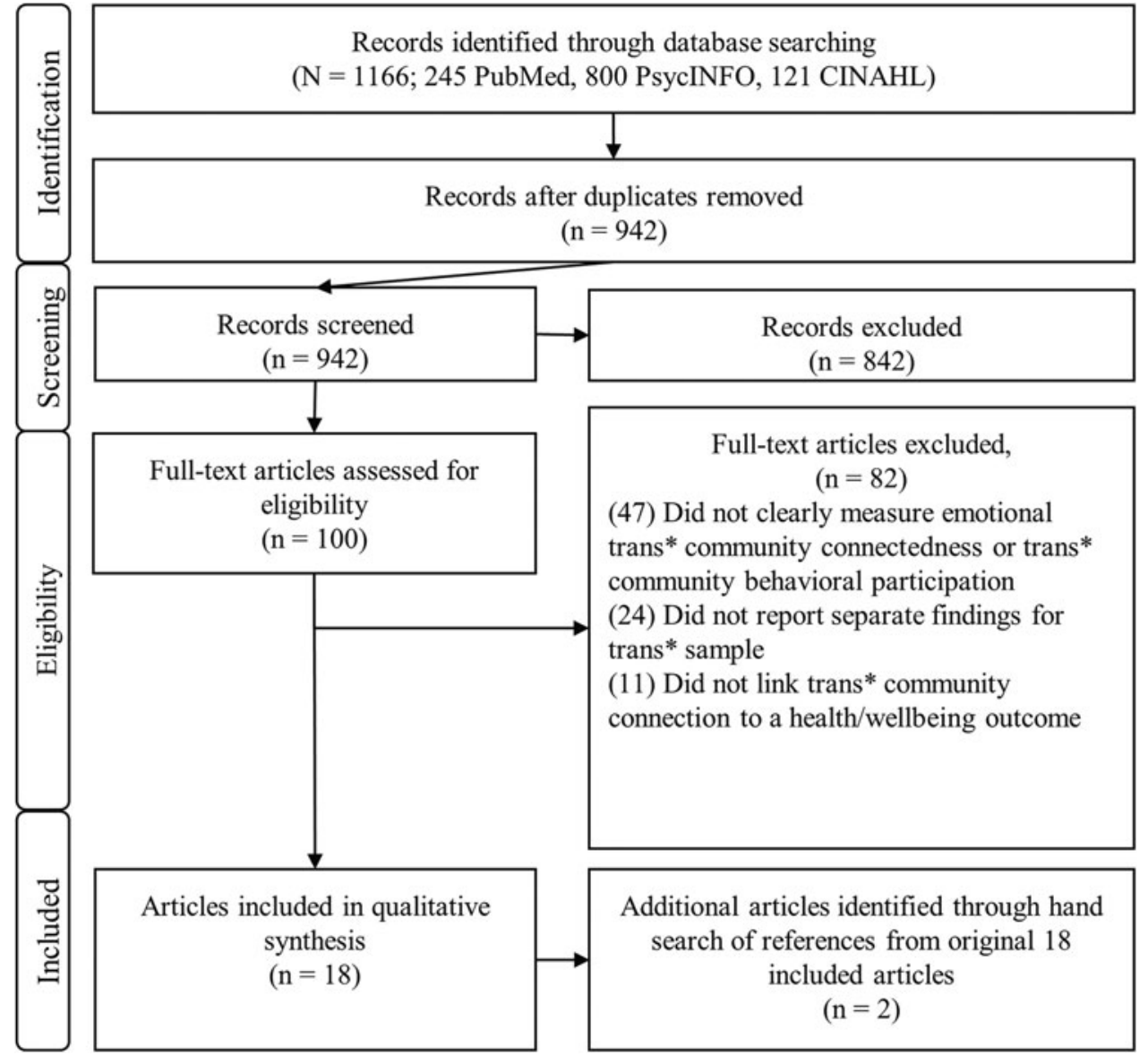

FIG. 1. The Preferred Reporting Items for Systematic Reviews and MetaAnalyses flow diagram.
Guatemala, ${ }^{28}$ and the United Kingdom. ${ }^{43}$ Data collection methods varied among studies and included self-administered in-person or online questionnaires $(n=7 / 20),{ }^{22,34-38,44}$ Photovoice focus groups $(n=1 / 20),{ }^{46}$ general focus groups $(n=2 / 20),{ }^{29,31}$ case studies $(n=1 / 20),{ }^{30}$ and individual interviews $(n=14 / 20) .^{28-33,36,38-43,45}$ Most studies used convenience sampling methods $(n=15 / 20), 22,31-36,38,39,41-46$ recruiting from trans*-associated venues such as support groups, health clinics, community-based organizations, and social media pages (Table 1).

Most articles reported eligibility requirements $(n=16 /$ 20). ${ }^{22,28-33,35-38,40-42,44,46}$ Two of the sixteen articles ${ }^{32,33}$ included transgender youth 15-25 years of age, while the remainder included only adults 18 years or older. ${ }^{22,28-31,35-}$ $38,40-42,44,46$ Ethnic/racial diversity varied among the studies, with $40 \%(n=8 / 20)^{22,30,33-36,39,44}$ of articles including samples comprised of over $50 \%$ White-identified participants, $30 \%(n=6 / 20)^{29,32,41,42,45,46}$ included only people of color, $15 \%(n=3 / 20)^{31,38,40}$ had a more equitable distribution of ethnicities/races, and $15 \%(n=3 / 20)^{28,37,43}$ failed to report race/ethnicity. Of the articles that had gender-diverse samples $(n=11 / 20),{ }^{22,31-35,37,39,40,43,44}$ only three articles examined nuances based on gender identity. ${ }^{22,34,35}$ Thirty-five percent $(n=7 / 20)^{28,29,38,41,42,45,46}$ of samples included transgender women only (not including comparison groups of gay/bisexual and heterosexual-identifying men who have sex with men), ${ }^{28}$ compared to $5 \%(n=1 / 20)^{36}$ of studies that focused on transgender men only (not including the comparison group of nontransgender gay and bisexual men). ${ }^{36}$ No studies focused exclusively on nonbinary people and one article failed to report gender identities of the full sample. ${ }^{30}$ Of the six articles with a quantitative component, inconsistent methods were applied to measure TCC and they did not capture both domains of TCC. ${ }^{2,34-38}$ See Table 2 for details regarding measurement.

\section{TCC and contextual associations}

The majority of studies reviewed were not designed specifically to identify the influence of TCC on outcomes; however, they did report findings related to TCC in addition to the main analysis. Few articles $(n=3 / 20)$ examined the association between demographic information (e.g., age, race/ethnicity, living environment, and gender identity variations) and TCC. ${ }^{22,32,33}$ Trans* youth (ages 15-25) from two small qualitative studies of high quality ( JBI quality score for qualitative studies $[Q L]=9 / 10^{32}$ and $10 / 10^{33}$ ) reported receiving more support for and affirmation of their gender identities and expressions from other trans* youth rather than from older trans* people. $^{32,33}$ Some individuals, regardless of age, felt a need to distance themselves from the community out of fear of being "outed" by affiliation, whereas others became more active in their communities despite the risk. ${ }^{32,33}$

Trans* youth (ages 15-24) from one of the studies $(N=13)$ that focused on behavioral participation reported that ethnic/racial subgroups within the trans* community were supportive of participants' multiple identities and "whole self", (e.g., supportive of both their gender and racial identities). ${ }^{32}$ 


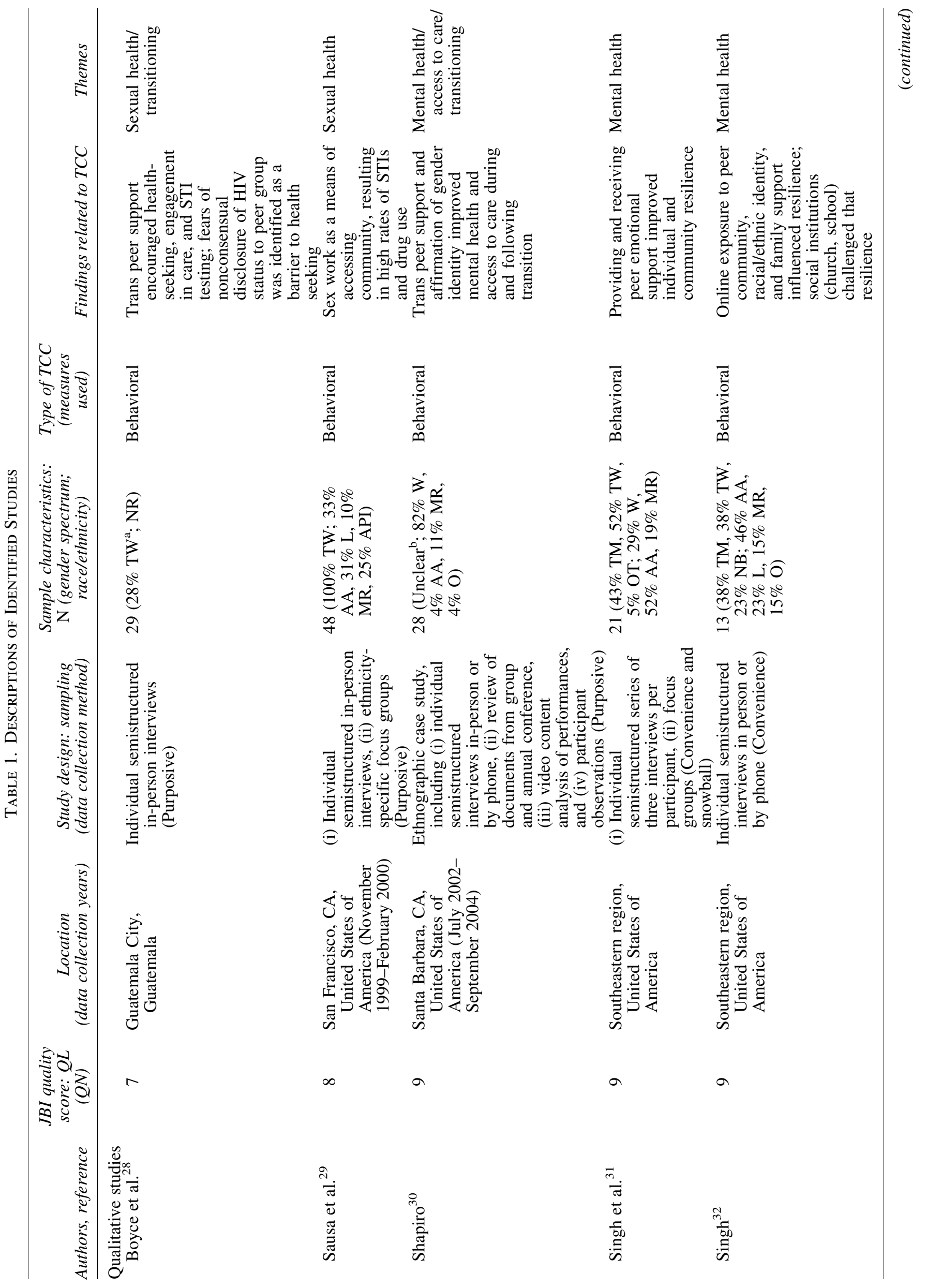




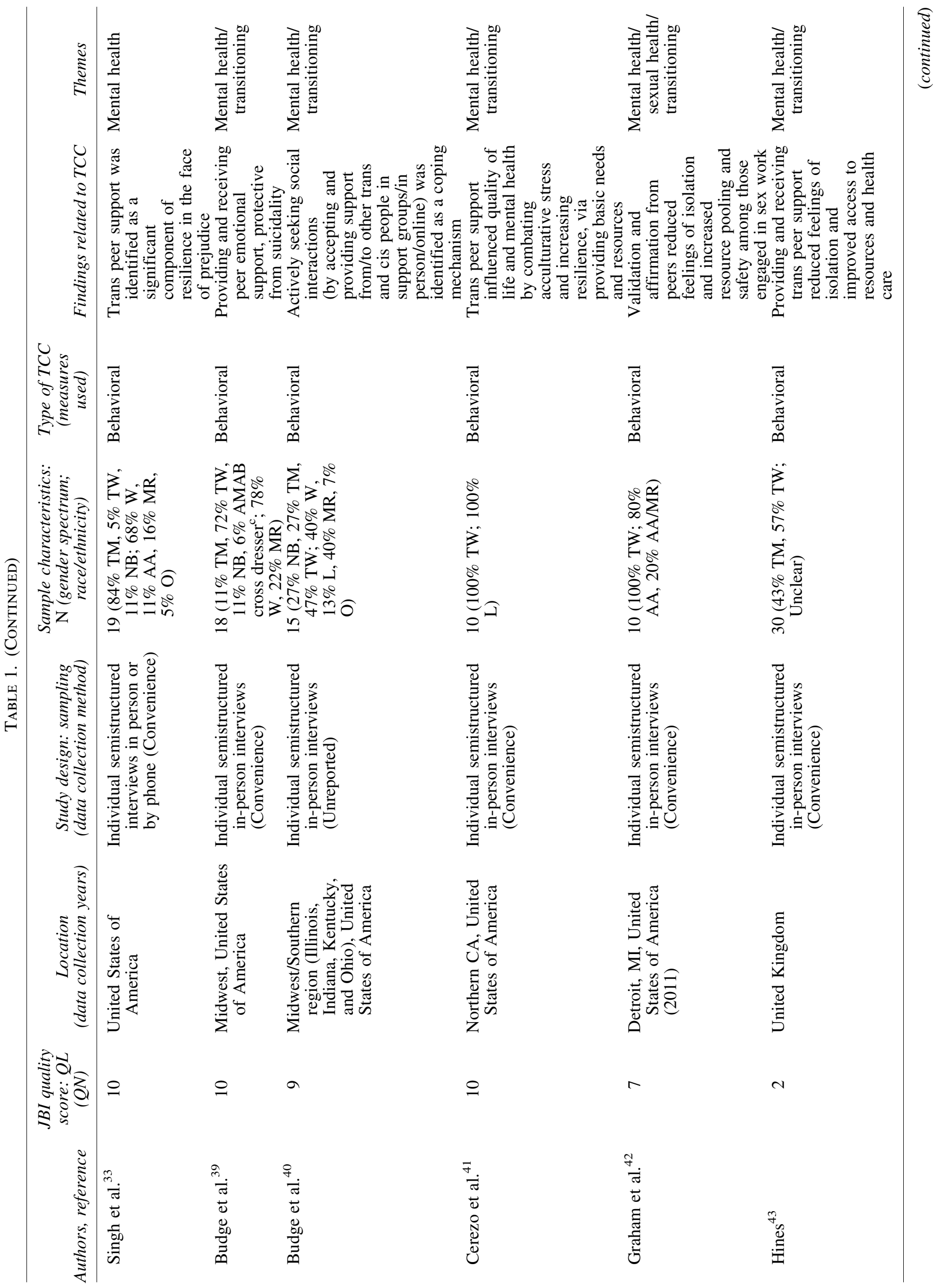




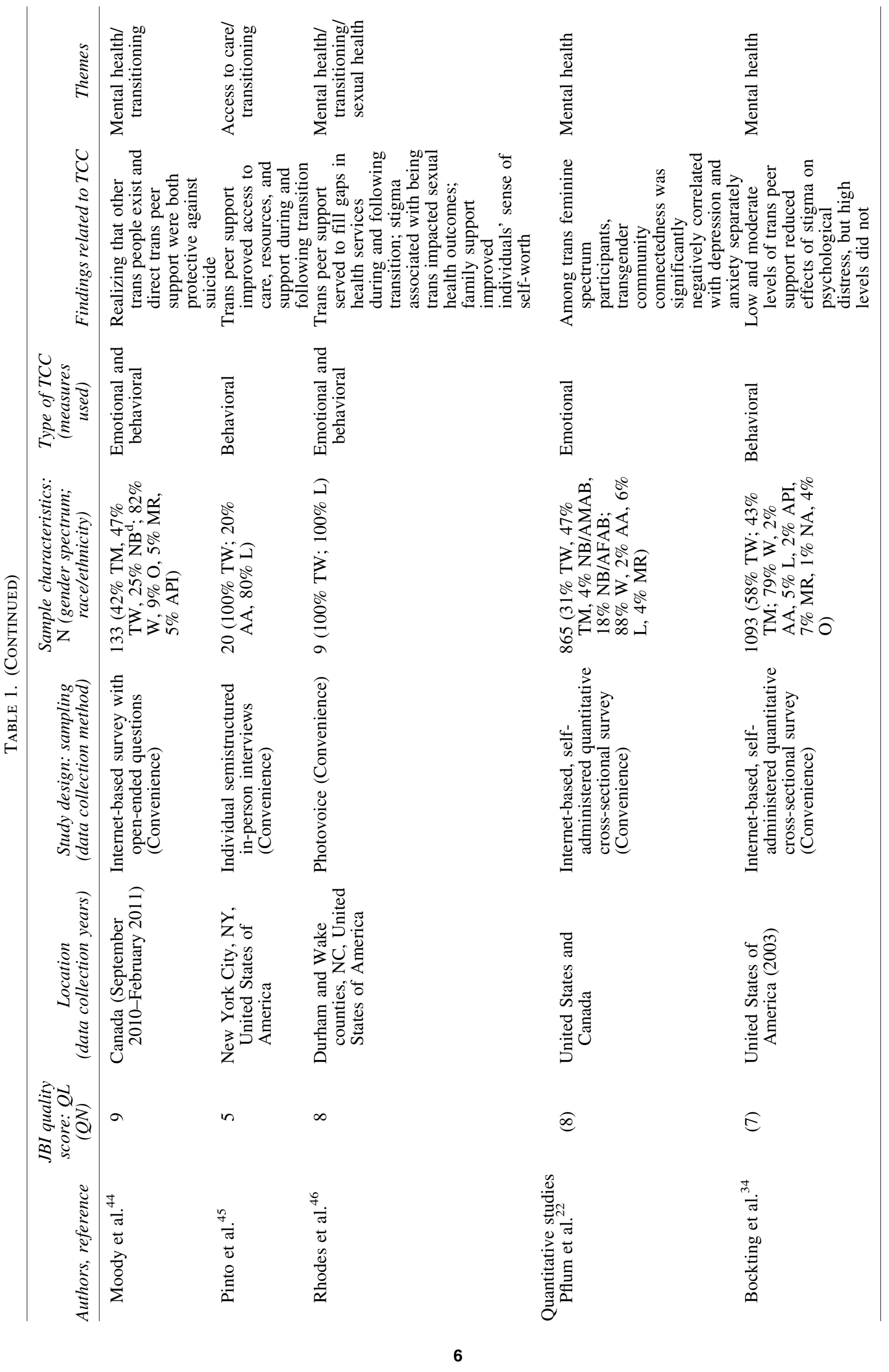




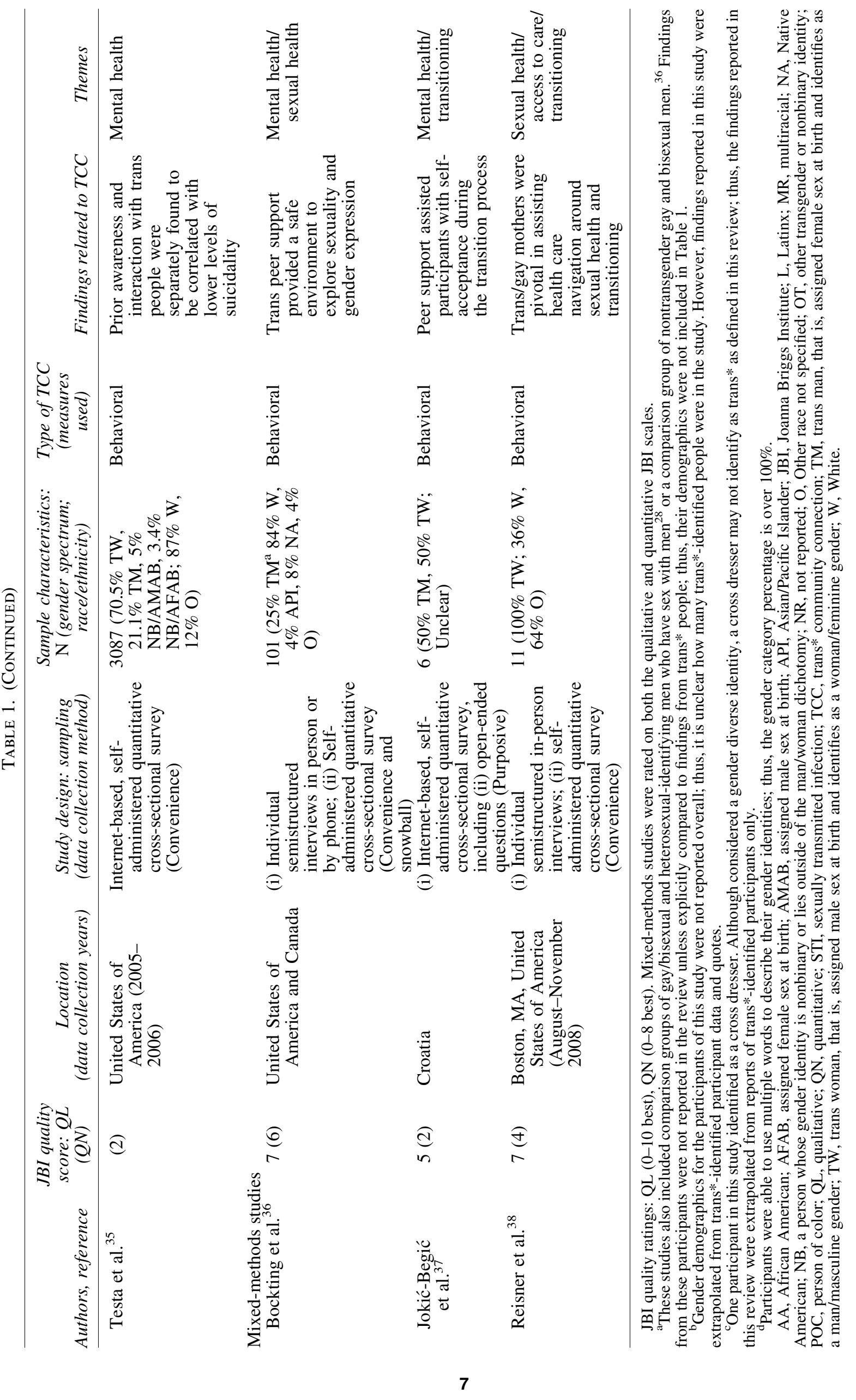


Table 2. Description of Quantitative Measures of Transgender Community Connection

\begin{tabular}{|c|c|c|c|c|}
\hline Authors, reference & Type of TCC & Measures used & Description of scale & Reliability/validity \\
\hline \multicolumn{5}{|l|}{ Quantitative studies } \\
\hline Pflum et al. ${ }^{22}$ & Emotional & $\begin{array}{l}\text { Gender Minority Stress and } \\
\text { Resilience Measure } \\
\text { [GMSR]; [Emotional] } \\
\text { Transgender Community } \\
\text { Connectedness Subscale }\end{array}$ & $\begin{array}{l}\text { Five item; five-point Likert } \\
\text { scale from strongly } \\
\text { disagree to strongly agree }\end{array}$ & $\begin{array}{l}\text { Cronbach's alpha } \\
0.61-0.93\end{array}$ \\
\hline Bockting et al. ${ }^{34}$ & Behavioral & $\begin{array}{l}\text { (1) "What portion of your } \\
\text { social time is spent with } \\
\text { transgender people?" (2) } \\
\text { "How often have you felt } \\
\text { like you were the only } \\
\text { transgender person in the } \\
\text { area that you live?" }\end{array}$ & $\begin{array}{l}\text { Seven-point Likert scale from } \\
\text { none of the time to all of } \\
\text { the time for Q1 and never } \\
\text { to always for Q2 }\end{array}$ & $\begin{array}{l}\text { Test-retest } \\
\text { reliability for } \\
n=20 \text { was } 0.87\end{array}$ \\
\hline Testa et al. ${ }^{35}$ & Behavioral & $\begin{array}{l}\text { "At what age did you first } \\
\text { meet another transgender } \\
\text { person?" }\end{array}$ & $\begin{array}{l}\text { Participants were coded as } \\
\text { having met another trans } \\
\text { person before identifying } \\
\text { as trans if the age span in } \\
\text { which they first met } \\
\text { another trans person was } \\
\text { before the age span in } \\
\text { which they first felt trans } \\
\text { themselves }\end{array}$ & NR \\
\hline \multicolumn{5}{|c|}{ Mixed-methods studies } \\
\hline Bockting et al. $^{36}$ & Behavioral & $\begin{array}{l}\text { Qualitative interviews } \\
\text { displayed theme, "The } \\
\text { Importance of } \\
\text { Community"; participants } \\
\text { reported sexual exploration } \\
\text { with others in the } \\
\text { transgender community }\end{array}$ & No quantitative scale reported & N/A \\
\hline Jokić-Begić et al. ${ }^{37}$ & Behavioral & $\begin{array}{l}\text { Asked the degree to which } \\
\text { participants were satisfied } \\
\text { with the support provided } \\
\text { to them during the 'sex } \\
\text { reassignment surgery' } \\
\text { process by trans individuals }\end{array}$ & No quantitative scale reported & N/A \\
\hline Reisner et al. ${ }^{38}$ & Behavioral & $\begin{array}{l}\text { "If we were to develop an } \\
\text { intervention or program for } \\
\text { male-to-female transgender } \\
\text { persons who perform sex } \\
\text { work, what do you see as } \\
\text { the most important areas on } \\
\text { which to focus our } \\
\text { efforts?" }\end{array}$ & No quantitative scale reported & N/A \\
\hline
\end{tabular}

NR, not reported; N/A, not applicable.

In addition, participants in this study reported that the Internet and social media were good places to connect with other youth who shared their racial/ethnic and gender identities. ${ }^{32}$ Participants found that interactions with racial/ethnic concordant trans* peers were affirming of both their racial/ethnic and trans* identities unlike among nonconcordant racial/ethnic peers. ${ }^{32}$ Concordant peers often became role models and a source of validation for racial and/or trans-specific life experiences. $^{32}$

A quantitative study of high quality (JBI quality score for quantitative studies $[\mathrm{QN}]=8 / 8 ; N=865$ trans* people), which focused on emotional connectedness, found no significant differences in mean scores for emotional connectedness when comparing people of color who were categorized as having gender identities on the trans feminine spectrum (TFS) (defined, in this specific study, as women or transgender women assigned male at birth, or nonbinary people assigned female at birth ${ }^{\mathrm{a}}$ ) and White TFS people. ${ }^{22}$ No other quantitative studies analyzed TCC by race/ethnicity. In the same study, there were no significant differences in emotional

${ }^{a}$ The TFS and TMS definitions in Pflum et al. ${ }^{22}$ are uncommon. Commonly, TFS and TMS are defined using sex assigned at birth and gender identity in the following manner: TFS is anyone assigned male at birth whose gender identity is more feminine than masculine (e.g., transgender woman and trans female); TMS is anyone assigned female at birth whose gender identity is more masculine than feminine (e.g., transgender man and trans male); and people with nonbinary identities that do not align with masculinity or femininity are categorized separately (e.g., agender and genderqueer). ${ }^{49}$ 
connectedness scores when compared by living environment using one-way analysis of variance for TFS participants ( $n=427 ; 45 \%$ urban, 33\% suburban, $14 \%$ small town, and $8 \%$ rural) and trans masculine spectrum (TMS) participants (defined, in this specific study, as men or transgender men assigned female at birth, or nonbinary people assigned male at birth; $n=438 ; 53 \%$ urban, $25 \%$ suburban, $15 \%$ small town, and $7 \%$ rural). ${ }^{22}$ No other studies examined the influence of geographic location on TCC.

\section{TCC and health outcomes}

Gender transitioning. Among 20 included articles, 12 $(60 \%)$ addressed the subject of gender transition related to TCC. ${ }^{28,30,37-46}$ Gender transition refers to the process whereby a person changes their gender expression from that associated with their sex assigned at birth to one that matches their current gender identity. ${ }^{50}$ Most articles in this category focused on behavioral participation with trans* peers only $(n=10$ / 12), ${ }^{28,30,37-43,45}$ while 2 of 12 covered behavioral participation and emotional connectedness. ${ }^{44,46}$ Most of these articles employed varying qualitative methods $(n=10 / 12)^{28,30,39-46}$ and 2 of 12 applied mixed methods ${ }^{37,38}$; see Table 1 for details regarding all included articles. Almost half of the samples in this group included people of different genders $(n=5 /$ 12). ${ }^{37,39,40,43,44} \mathrm{Six}$ of the 12 articles included only transgender women ${ }^{28,38,41,42,45,46}$ and the final study did not report gender identities for all participants $(n=1 / 12) .^{30}$

Although quality varied (see Table 1 for quality scores), several articles reported that TCC helped to improve healthrelated behaviors, such as setting appointments to receive hormones. ${ }^{28,30,38,43,45}$ Participants from three qualitative studies of high quality (ranging $Q L=9 / 10-10 / 10$ ) indicated that providing emotional support or guidance to others during their gender transition improved the mental health of the provider of said support. ${ }^{31,39,40}$ However, the most common theme noted among the majority of these studies was that receiving emotional support and validation from trans* peers helped trans* people feel less alone and aided in improving mental health related to gender transition for the receiver. ${ }^{28,30,37,39,40,42-46}$

Mental health. TCC was found to be linked to mental health/wellbeing and was the topic of the majority of articles identified $(n=16 / 20) .^{22,30-37,39-44,46}$ Mental health/wellbeing topics included emotional resilience $(n=3 / 16),{ }^{31-33}$ suicidality $(n=3 / 16),{ }^{35,39,44}$ undefined general mental health $(n=3 /$ $16),{ }^{30,36,41}$ isolation $(n=2 / 16),{ }^{42,43}$ depression $(n=2 / 16),{ }^{22,34}$ anxiety $(n=2 / 16),{ }^{22,34}$ sense of self-worth $(n=1 / 16),{ }^{46}$ positive coping behaviors $(n=1 / 16),{ }^{46}$ and self-acceptance $(n=1 / 16) .{ }^{37}$ Most of these articles focused on behavioral participation only $(n=13 / 16),{ }^{30-37,39-43}$ whereas one explored emotional connectedness only $(n=1 / 16)^{22}$ and two covered both behavioral participation and emotional connectedness $(n=2 / 15){ }^{44,46}$

The largest number of articles in this area employed qualitative methods $(n=11 / 16),{ }^{30-33,39-44,46}$ with far fewer using quantitative methods $(n=3 / 16),{ }^{22,34,35}$ and two using a mix of both quantitative and qualitative methods. ${ }^{36,37}$ Most samples included multiple subgroups of trans* people $(n=11 /$ $16), 22,31-35,37,39,40,43,44$ while three studies had homogeneous samples that only included transgender women. ${ }^{41,42,46}$
A large $(N=1093)$, predominantly White $(79 \%)$, sample of transgender men and transgender women participated in a high-quality $(\mathrm{QN}=7 / 8)$ cross-sectional survey study, which revealed that TCC, through behavioral participation, moderated the association between stigma and psychological distress at low and moderate, but not high levels of trans* peer support, which suggests a ceiling effect (referring to the point at which the amount of behavioral participation no longer affects the psychological distress noted) that was not explored elsewhere. ${ }^{34}$ A similarly designed high-quality study $(\mathrm{QN}=8 / 8$; $N=865$, including transgender men, transgender women, and nonbinary people; $88 \%$ White) found that emotional connectedness was significantly negatively correlated with symptoms of depression and anxiety for TFS participants, but not for TMS participants. ${ }^{22}$

As seen among articles focused on gender transition, ${ }^{28,30,37,39,40,42-46}$ qualitative interviews from several studies of trans* people showed that trans* peer support had a positive relationship with participants' emotional experiences and helped reduce suicidality through the provision of emotional support. ${ }^{31,39,44}$ Specifically, one of these articles reported that it was typically trans* peers who provided support when participants were suicidal. ${ }^{31}$ Participants from another study reported being known and respected in the trans* community for not acting on suicidal intentions. ${ }^{43}$

TCC has been linked with the development of coping strategies and emotional resilience. ${ }^{31,33,39,40}$ Budge et al. $(\mathrm{QL}=9 /$ 10) used grounded theory among a sample of 15 trans* people to identify facilitative and avoidant coping behaviors throughout gender transition and found that actively seeking social interactions with the trans* community, attending support groups, and relying on trans* people for help were found to be facilitative coping behaviors. ${ }^{40}$ Another high-quality study $(\mathrm{QL}=10 / 10)$ with 19 trans* youth reported that some trans* support groups were either not tailored to their other social identities (e.g., race/ethnicity) or focused too highly on medical transitioning. ${ }^{33}$ Trans* people also reported that being able to provide and receive guidance and support from trans* peers provided them with a sense of pride, meaning, success, and increased emotional resilience. ${ }^{31,33,39}$

Sexual health. Among 20 included articles, 6 articles $(30 \%)$ addressed the subject of sexual health in relation to TCC, specifically related to behavioral participation in the trans* community. ${ }^{28,29,36,38,42,46}$ Most of these articles employed qualitative methods $(n=4 / 6),{ }^{28,29,42,46}$ while the remainder employed both quantitative and qualitative methods $(n=2 / 6) .^{36,38}$ One of the six studies had only transgender men $^{36}$ (not including the comparison group of nontransgender gay and bisexual men) and the remaining studies only included transgender women ( $n=5 / 6$, not including comparison groups of gay/bisexual and heterosexual-identifying men who have sex with men); ${ }^{28}$ see Table $1 .{ }^{28,29,38,42,46}$

Many trans* people who disclose their gender identity to their family experience rejection, which can result in social vulnerability to exploitation. ${ }^{38,42}$ This biological family void is often replaced by "chosen families" (e.g., selfidentified family members who are not related by blood or marriage) comprised of lesbian, gay, bisexual, transgender, and queer (LGBTQ+) peers. ${ }^{29,38,42}$ One study (QN $=6 / 8$; $\mathrm{QL}=7 / 10)$ with a sample of transgender men who were predominantly White $(n=21 / 25)$ found that interaction with trans* 
peers provided a safe environment to explore one's sexuality and gender expression. ${ }^{36}$ Meanwhile, in some instances, a gender questioning person's first access to the visible community of transgender women has been through street engaged transgender women who trade sex. ${ }^{29}$ One participant from this qualitative study $(\mathrm{QL}=8 / 10)$ noted that sex work was almost like a rite of passage for young transgender women and was viewed as a way to connect with the trans* community. ${ }^{29}$ In some instances, chosen families and trans* mothers (trans* people who take on a maternal role for nonbiological kin) have introduced transgender women to sex work (e.g., trading sex for money, housing, and/or safety); however, transgender women who trade sex often report doing so to offset a lack of income and shelter due to employment and housing discrimination. ${ }^{28,29,38}$ Trans* mothers and trans* peers in these situations have provided support to newly transitioning trans* people by teaching them how to find sex work clients and care for their sexual health, and by sharing shelter and resources. ${ }^{29,38,42}$

However, sex work environments can be dangerous and competitive, stemming from the limited number of sex work clients. $^{28,51}$ This competition affected the lives of a few participants from one qualitative study $(\mathrm{QL}=7 / 10)$ of transgender women $(N=8)$ who, in turn, expressed concerns about confidentiality while attending trans*-specific clinic days. ${ }^{28}$ These participants reported a fear of trans* peers starting rumors or disclosing the participant's HIV status against their will to potential sex work clients or peers. ${ }^{28}$ In contrast, a common theme from this study found that some participants felt a sense of belonging (emotional connectedness) at the clinic in their area because they provided a designated clinic schedule for trans* sex workers with knowledgeable staff and free services. ${ }^{28}$

Access to care. Among 20 included articles, 3 (15\%) assessed relationships between access to care and TCC, specifically behavioral participation in the trans* community. ${ }^{30,38,45}$ Two articles used qualitative methods, ${ }^{30,45}$ while one article used mixed methods. ${ }^{38}$ Samples were limited to transgender women and were comprised of more than $50 \%$ people of color. ${ }^{38,45}$ In one study, the gender identities were not reported clearly, and over $80 \%$ of the sample was White. ${ }^{30}$ All articles provided context for how community engagement encouraged transgender individuals in urban environments to seek out health care for gender transition-related needs, sexually transmitted infection testing, and preventive care. ${ }^{30,38,45}$

\section{Discussion}

Across all of the articles reviewed, TCC was linked with several positive health outcomes, including improved mental health, increased connection to care, increased comfort with the exploration of sexual and gender identities, and informed gender transition. Unfortunately, TCC was not the primary focus of the majority of articles; therefore, the breadth and depth of results (both quantitative and qualitative) were minimal. This lack of detail and the limited number of articles addressing a broad range of relationships to TCC made data synthesis challenging.

Some specific findings may guide and inform future research. Variations in the strength of the effect of TCC on symptoms of depression and anxiety were noted between TFS and TMS participants in one quantitative study ${ }^{22}$; how- ever, this study used unique categorizations for TFS and TMS that are not reflective of many studies in trans* health research. ${ }^{52,53}$ This study defined TFS generally as women or transgender women assigned male at birth, or nonbinary people assigned female at birth, and TMS as men or transgender men assigned female at birth, or nonbinary people assigned male at birth. ${ }^{22}$ This unique categorization makes the findings from Pflum et al.'s study unclear; however, this article was one of only three included articles that looked at gender differences at all. ${ }^{22,34,35}$ Findings from those studies suggest that there may be differences in how people of different gender identities interact with the trans* community and how influential TCC may be; thus, more research with gender diverse samples using consistent measurement is needed to better understand these potential differences. ${ }^{22,34,35}$

Another finding from one high-quality quantitative study with transgender women stood out among the research and suggested a potential ceiling effect-referring to the point at which the amount of behavioral participation no longer affected the psychological distress noted. ${ }^{34}$ This potential ceiling effect is understudied, and it is unclear what could be influencing this phenomenon. One may infer that those with the highest behavioral participation scores could be exposed to additional stigma or other factors affecting psychological distress, such as being publicly "outed" by association with others who are visibly trans*. ${ }^{17,32-34}$ More research is needed to better understand this effect.

TCC was also associated with exposure to sex work and may deter transgender women from getting sexual health testing and treatment due to fear of being observed at specialized clinics and subsequent loss of confidentiality among peers. ${ }^{28}$ This finding regarding a potential loss of confidentiality is troubling, especially because there are strong calls for trans* community representation in health care settings. ${ }^{50}$ Many clinics are recruiting trans* people as staff, and this may further deter health-seeking if proper confidentiality training and community trust are not assured. ${ }^{18}$ In addition, participants reported that the clinic provided a trans*-specific clinic day, which caused concern that their trans* peers (who were also patients attending the clinic) would disclose their presumed health information to others in the community. ${ }^{28}$ This finding may be related to a heightened awareness of other trans* peers due to the modest size of many local trans* communities and the potential competition for resources as noted previously.

Intersectionality-the experiences of oppression and/or privilege experienced by a person based on their multiple, co-occurring identities (e.g., race, ethnicity, sexual orientation, and gender identity) - is an important consideration for the examination of TCC and related health outcomes. ${ }^{54}$ The effects of intersectional identities are far-reaching, influencing interactions with health systems and health professionals, and whether one has access to care, ultimately affecting health outcomes. ${ }^{5-57}$ The research included in this review alluded to the intersectional nature of ethnicity/race, gender, geographic location (urban/rural), and age as important to consider when discussing TCC; however, the included articles (even those that alluded to the effect of additional social variables) failed to examine the nuanced relationship between health outcomes and TCC through an intersectional lens. For example, geographic location may affect the size of the community, and the accessibility of 
community members or community, thereby affecting TCC. In addition, health disparities may be heightened in less populated areas with reduced access to trans* health providers. However, only one article examined the effects of geographic location and found no significant difference in TCC by location, most likely related to the small sample sizes within each group-highlighting the need for trans* research with more robust sample sizes. ${ }^{22}$

Similar findings of no difference in TCC were found regarding race/ethnicity in the aforementioned study. ${ }^{22}$ Racial/ethnic communities may have social norms that could affect TCC and health outcomes differently. Connections to multiple different communities may provide additional protections or opportunities for harm ${ }^{32,33}$; for example, race- or ethnicity-based community may provide protection from racism, but may perpetuate cisgenderism, or vice versa. Regardless, Pflum et al.'s study had a small sample of people of color and was unable to look at nuances between subgroups, and did not find any differences between White participants and participants of color. ${ }^{22}$ This study also did not take an intersectional lens to extrapolate any nuance in findings, while considering multiple identities. $^{22}$

Often, articles included in this review that used rigorous methods and study designs did not recruit large, diverse samples based on ethnicity/race and gender identity; therefore, the extent to which intersectionality, identity, and demographic factors influence TCC and health disparities remains unclear. This issue calls for researchers to strive to recruit more diverse and representative samples to investigate whether differences are significant and nuances impact health outcomes for different groups. The overrepresentation of transgender women in many studies also may affect how the larger trans* population is understood and how systems and programs are developed by assuming uniformity in experience. More research with transgender men and nonbinary people is needed, and future research should consider the implications of power and oppressive differentials and their effects on interacting subcommunities of trans* people.

As mentioned previously, many of the included articles were narrow in scope and depth concerning the investigation of TCC and health outcomes. Among the included articles, topic areas included (i) gender transitioning, (ii) mental health, (iii) sexual health, and (iv) access to care. The majority of the research focused on mental health $(80 \%)$ and the gender transition $(60 \%)$ period, which may work to perpetuate historical pathological models that viewed gender nonconformity as a symptom of psychopathology. Such pathologizing models have since been removed from the Diagnostic and Statistical Manual of Mental Disorders (DSM), and the International Statistical Classification of Diseases and Related Health Problems (ICD), as has the stigmatizing diagnosis of Gender Identity Disorder. ${ }^{58,59}$ The DSM, for example, now includes the diagnosis of gender dysphoria, which emphasizes that the focus of treatment is on the dysphoria, not the gender identity, while the ICD has named this entity "Gender Incongruence" and does not classify it as a mental disorder. ${ }^{58,59}$

Much research refutes earlier notions that trans* people are inherently mentally ill by identifying socially produced disparities in exposure to oppression, stigma, and violence, as well as associated health outcomes. This early emphasis on mental health combined with influential funding streams incentivizing HIV-related inquiry has skewed trans* health research resulting in minimal physical health coverage beyond HIV and mental health contexts. Overall, further research is needed to investigate additional physical health outcomes that could be influenced by TCC.

The articles in this review suggest that TCC increases health care access; however, the duration of this effect was not addressed. There are not enough data to determine whether transgender people continue to seek services regularly or if the effect dissipates over time. No evaluation of treatment-related behaviors or medication adherence was made. Further examination of how TCC may be related to treatment and medication adherence for those with chronic diseases is an important area for future research.

The validity and reliability of many current measures assessing TCC and behavioral participation have yet to be adequately psychometrically evaluated with trans* samples. The included articles also did not adequately measure both behavioral participation and community connectedness; thus, the findings often do not provide a holistic picture of TCC. In addition, the recruitment of participants from trans-focused outlets and by snowball sampling may have produced a bias toward people who are more connected to the transgender community, and therefore limits the generalizability of the findings.

Finally, the current TCC literature presents mostly crosssectional data, which limit causal inferences being made about the relationship between community connectedness and potential health outcomes. More robust longitudinal data would establish whether there are stronger connections and assist in the identification of modifiable factors to improve the impact of TCC. In addition, more mixed methods and rigorous sampling for future studies are needed to provide a comprehensive understanding of nuances within subgroups of trans* people.

\section{Strengths and limitations}

The dual reviewer systematic identification and appraisal of the included articles is a considerable strength of the review. Data extraction was completed solely by the first author; however, this limitation was addressed by a $25 \%$ audit of extracted data performed by an additional team member. The standardized measures used in the appraisal process increase the reliability of the review; however, the quality appraisal method to assess mixed-methods articles was not ideal because of a lack of tools available to assess mixed-methods articles. Thus, appraisals for mixedmethods articles reviewed should be considered within this limitation. In addition, relevant articles may have been missed due to the exclusion of articles published before 2007, gray literature, dissertations, and quantitative articles on processes or measure development. Although articles had to be published from January 1, 2007, to November 1, 2017, several articles reported findings from data collected many years before 2007. Thus, findings from these more dated sources may be less representative of the current community influences within trans* communities. However, this review provides a meaningful synthesis of the current state of TCC peer-reviewed research in attempts to guide further investigation. 


\section{The way forward: recommendations}

This systematic research review highlights the main findings despite the inconsistencies within the research addressing associations between TCC and trans* health and wellbeing. The quality of the articles varied widely. Results referencing subgroup analysis by gender and ethnicity/race were often unreliable due to sampling size and measurement inadequacies. Future development and testing of new and current instruments to accurately measure TCC among different subgroups will improve the quality and rigor of the data that emerge. With valid and reliable measures, the next step would be to design more longitudinal studies to allow for causal effects to be evaluated. Recruitment of participants from non-trans*-specific environments will allow researchers to capture a broader sample of people with high and low TCC and that is more representative of different races/ ethnicities in all subgroups of trans* people. Moreover, rigorous purposive sampling methods may assist in recruiting more ethnically/racially and gender diverse samples to provide a better understanding of the nuances affecting different subgroup communities. ${ }^{60,61}$

Finally, the current literature examining TCC has focused overwhelmingly on the gender transition period, which fails to acknowledge potential influences on health before and following transition. Health care professionals have an obligation to address health disparities throughout the lifespan, especially for trans* people who are disproportionately exposed to violence and stigma. It is paramount that health professionals and researchers work together to better understand health disparities affecting trans* people and develop interventions to reduce barriers to care, ultimately contributing to the improvement of health among trans* people. Future research should expand upon existing literature and investigate how to leverage TCC to improve physical health, mental health, and general health care access and utilization.

\section{Conclusion}

Although there is substantial literature describing a predominantly positive relationship between TCC and the health and wellbeing of trans* people, opportunities remain. This review establishes that the measurement of TCC and its application are broad and inconsistent. The development of more consistent measures of TCC could provide effective tools in future intervention-based studies leveraging TCC to improve health outcomes for trans* people.

\section{Acknowledgment}

Informal mentorship for this article was received from Sarah Allgood, PhD, RN.

\section{Disclaimer}

This review was previously presented in the following format: Ford A, Clark K, Robinson K, Noorani T, and Poteat T. Transgender Community Connection and Health Outcomes: A systematic research review. American Public Health Association Annual Meeting \& Expo, San Diego, CA, 2018 (Poster).

\section{Author Disclosure Statement}

No competing financial interests exist.

\section{Funding Information}

Indirect funding for this work came from the Robert Wood Johnson Foundation Future of Nursing Scholars program.

\section{References}

1. Ansara YG, Hegarty P: Cisgenderism in psychology: Pathologising and misgendering children from 1999 to 2008. Psychol Sex 2011;3:137-160.

2. Ansara YG, Hegarty P: Methodologies of misgendering: Recommendations for reducing cisgenderism in psychological research. Fem Psychol 2014;24:259-270.

3. Tebbe EA, Moradi B, Ege E: Revised and abbreviated forms of the Genderism and Transphobia Scale: Tools for assessing anti-trans prejudice. J Couns Psychol 2014; 61:581-592.

4. White Hughto JM, Reisner SL, Pachankis JE: Transgender stigma and health: A critical review of stigma determinants, mechanisms, and interventions. Soc Sci Med 2015;147: 222-231.

5. Baral SD, Poteat T, Strömdahl S, et al.: Worldwide burden of HIV in transgender women: A systematic review and meta-analysis. Lancet Infect Dis 2013;13:214-222.

6. Coulter RWS, Mair C, Miller E, et al.: Prevalence of pastyear sexual assault victimization among undergraduate students: Exploring differences by and intersections of gender identity, sexual identity, and race/ethnicity. Prev Sci 2017; 18:726-736.

7. Langenderfer-Magruder L, Walls NE, Kattari SK, et al.: Sexual victimization and subsequent police reporting by gender identity among lesbian, gay, bisexual, transgender, and queer adults. Violence Vict 2016;31:320-331.

8. Nuttbrock L, Hwahng S, Bockting W, et al.: Psychiatric impact of gender-related abuse across the life course of maleto-female transgender persons. J Sex Res 2010;47:12-23.

9. Reisner SL, White Hughto JM, Gamarel KE, et al.: Discriminatory experiences associated with posttraumatic stress disorder symptoms among transgender adults. J Couns Psychol 2016;63:509-519.

10. Haas AP, Rodgers PL, Herman JL: Suicide attempts among transgender and gender non-conforming adults: Findings of the national transgender discrimination survey. 2014. Available at https://queeramnesty.ch/docs/AFSP-WilliamsSuicide-Report-Final.pdf Accessed August 2, 2017.

11. Nuttbrock L, Bockting W, Rosenblum A, et al.: Gender abuse, depressive symptoms, and HIV and other sexually transmitted infections among male-to-female transgender persons: A three-year prospective study. Am J Public Health 2013;103:300-307.

12. Grant JM, Mottet LA, Tanis J, et al.: Injustice at Every Turn: A Report of the National Transgender Discrimination Survey. 2011. Washington, DC, National Center for Transgender Equality and National Gay and Lesbian Task Force.

13. Guadalupe-Diaz XL, Jasinski J: "I wasn't a priority, I wasn't a victim": Challenges in help seeking for transgender survivors of intimate partner violence. Violence Against Women 2017;23:772-792.

14. GLBT Domestic Violence Coalition and Jane Doe, Inc.: Shelter/housing needs for gay, lesbian, bisexual and transgender (GLBT) victims of domestic violence. Analysis of public hearing testimony. October 27, 2005. Boston, MA, Massachusetts State House. Available at http://ncdsv.org/ images/ShelterHousingNeedsforGLBTVictimsDV.pdf Accessed October 30, 2017. 
15. Reck J: Homeless gay and transgender youth of color in San Francisco: "No one likes street kids"-Even in the Castro. J LGBT Youth 2009;6:223-242.

16. Poteat T, German D, Kerrigan D: Managing uncertainty: A grounded theory of stigma in transgender health care encounters. Soc Sci Med 2013;84:22-29.

17. James SE, Herman JL, Rankin S, et al.: The Report of the 2015 U.S. Transgender Survey. 2016. Washington, DC, National Center for Transgender Equality.

18. Sherman AD: TransConnect: A mixed methods study of pathways to health for Black transgender women who have survived violence (doctoral dissertation). Baltimore, MD: Johns Hopkins University, 2019.

19. Meyer IH, Dean L: Internalized homophobia, intimacy, and sexual behavior among gay and bisexual men. In: Psychological Perspectives on Lesbian and Gay Issues, Vol. 4. Stigma and Sexual Orientation: Understanding Prejudice Against Lesbians, Gay Men, and Bisexuals. Edited by Herek GM. Thousand Oaks, CA: Sage Publications, Inc., 1998, pp 160-186.

20. Meyer IH: Prejudice, social stress, and mental health in lesbian, gay, and bisexual populations: Conceptual issues and research evidence. Psychol Bull 2003;129:674-697.

21. Hendricks ML, Testa RJ: A conceptual framework for clinical work with transgender and gender nonconforming clients: An adaptation of the Minority Stress Model. Prof Psychol Res Pract 2012;43:460-467.

22. Pflum SR, Testa RJ, Balsam KF, et al.: Social support, trans community connectedness, and mental health symptoms among transgender and gender nonconforming adults. Psychol Sex Orientat Gend Divers 2015;2:281-286.

23. Bradford J, Reisner SL, Honnold JA, Xavier J: Experiences of transgender-related discrimination and implications for health: Results from the Virginia Transgender Health Initiative Study. Am J Public Health 2013;103:1820-1829.

24. Pan ML: Preparing Literature Reviews: Qualitative and Quantitative Approaches, 5th ed. New York: Routledge, 2016.

25. Cavalcante A: "I did it all online": Transgender identity and the management of everyday life. Crit Stud Media Commun 2016;33:109-122.

26. Covidence systematic review software. Veritas health innovation, Melbourne, Australia. 2019. Available at https:// covidence.org/home Accessed June 4, 2019.

27. Liberati A, Altman DG, Tetzlaff J, et al.: The PRISMA statement for reporting systematic reviews and metaanalyses of studies that evaluate health care interventions: Explanation and elaboration. J Clin Epidemiol 2009;62: e1-e34.

28. Boyce S, Barrington C, Bolaños H, et al.: Facilitating access to sexual health services for men who have sex with men and male-to-female transgender persons in Guatemala City. Cult Health Sex 2012;14:313-327.

29. Sausa LA, Keatley J, Operario D: Perceived risks and benefits of sex work among transgender women of color in San Francisco. Arch Sex Behav 2007;36:768-777.

30. Shapiro E: Drag kinging and the transformation of gender identities. Gend Society 2007;21:250-271.

31. Singh AA, Hays DG, Watson LS: Strength in the face of adversity: Resilience strategies of transgender individuals. J Couns Dev 2011;89:20-27.

32. Singh AA: Transgender youth of color and resilience: Negotiating oppression and finding support. Sex Roles 2013;68: 690-702.
33. Singh AA, Meng SE, Hansen AW: "I am my own gender": Resilience strategies of trans youth. J Couns Dev 2014;92: 208-218.

34. Bockting WO, Miner MH, Swinburne Romine RE, et al.: Stigma, mental health, and resilience in an online sample of the US transgender population. Am J Public Health 2013;103:943-951.

35. Testa RJ, Jimenez CL, Rankin S: Risk and resilience during transgender identity development: The effects of awareness and engagement with other transgender people on affect. J Gay Lesbian Ment Health 2014;18:31-46.

36. Bockting W, Benner A, Coleman E: Gay and bisexual identity development among female-to-male transsexuals in North America: Emergence of a transgender sexuality. Arch Sex Behav 2009;38:688-701.

37. Jokić-Begić N, Lauri Korajlija A, Jurin T: Psychosocial adjustment to sex reassignment surgery: A qualitative examination and personal experiences of six transsexual persons in Croatia. Scientific World J 2014;2014:960745. eCollection 2014.

38. Reisner SL, Mimiaga MJ, Bland S, et al.: HIV risk and social networks among male-to-female transgender sex workers in Boston, Massachusetts. J Assoc Nurses AIDS Care 2009;20:373-386.

39. Budge SL, Katz-Wise SL, Tebbe EN, et al.: Transgender emotional and coping processes: Facilitative and avoidant coping throughout gender transitioning. Couns Psychol 2013;41:601-647.

40. Budge SL, Chin MY, Minero LP: Trans individuals' facilitative coping: An analysis of internal and external processes. J Couns Psychol 2017;64:12-25.

41. Cerezo A, Morales A, Quintero D, Rothman S: Trans migrations: Exploring life at the intersection of transgender identity and immigration. Psychol Sex Orientat Gend Divers 2014;1:170-180.

42. Graham LF, Crissman HP, Tocco J, et al.: Interpersonal relationships and social support in transitioning narratives of Black transgender women in Detroit. Int $\mathrm{J}$ Transgend 2014;15:100-113.

43. Hines S: Transgendering care: Practices of care within transgender communities. Crit Soc Policy 2007;27:462486.

44. Moody C, Fuks N, Peláez S, Smith NG: "Without this, I would for sure already be dead": A qualitative inquiry regarding suicide protective factors among trans adults. Psychol Sex Orientat Gend Divers 2015;2:266-280.

45. Pinto RM, Melendez RM, Spector AY: Male-to-female transgender individuals building social support and capital from within a gender-focused network. J Gay Lesbian Soc Serv 2008;20:203-220.

46. Rhodes SD, Alonzo J, Mann L, et al.: Using photovoice, Latina transgender women identify priorities in a new immigrant-destination state. Int J Transgend 2015;16: 80-96.

47. Lockwood C, Munn Z, Porritt K: Qualitative research synthesis: Methodological guidance for systematic reviewers utilizing meta-aggregation. Int J Evid Based Healthc 2015;13: 179-187.

48. Moola S, Munn Z, Tufanaru C, et al.: Chapter 7: Systematic reviews of etiology and risk. In: Joanna Briggs Institute Reviewer's Manual. Edited by Aromataris E, Munn Z. Adelaide, Australia: The Joanna Briggs Institute, 2017. Available at https://reviewersmanual.joannabriggs.org/ Accessed September 28, 2019. 
49. Reisner SL, Radix A, Deutsch MB: Integrated and genderaffirming transgender clinical care and research. J Acquir Immune Defic Syndr 2016;72:S235-S242.

50. Coleman E, Bockting W, Botzer M, et al.: Standards of care for the health of transsexual, transgender, and gender-nonconforming people, version 7. Int J Transgend 2012;13:165-232.

51. Bith-Melander P, Sheoran B, Sheth L, et al.: Understanding sociocultural and psychological factors affecting transgender people of color in San Francisco. J Assoc Nurses AIDS Care 2010;21:207-220.

52. dickey lm, Reisner SL, Juntunen CL: Non-suicidal selfinjury in a large online sample of transgender adults. Prof Psychol Res Pract 2015;46:3-11.

53. Hwahng SJ, Nuttbrock L: Adolescent gender-related abuse, androphilia, and HIV risk among transfeminine people of color in New York City. J Homosex 2014;61:691-713.

54. de Vries KM: Intersectional identities and conceptions of the self: The experience of transgender people. Symb Interact 2012;35:49-67.

55. Jaffee KD, Shires DA, Stroumsa D: Discrimination and delayed health care among transgender women and men: Implications for improving medical education and health care delivery. Med Care 2016;54:1010-1016.

56. Williams DR, Priest N, Anderson NB: Understanding associations among race, socioeconomic status, and health: Patterns and prospects. Health Psychol 2016;35:407-411.

57. Williams DR, Mohammed SA, Leavell J, Collins C: Race, socioeconomic status, and health: Complexities, ongoing challenges, and research opportunities. Ann N Y Acad Sci 2010;1186:69-101.

58. American Psychiatric Association: Diagnostic and Statistical Manual of Mental Disorders (DSM- $\left.5^{\circledR}\right)$. Arlington, VA: American Psychiatric Association Publishing, 2013.

59. Reed GM, Drescher J, Krueger RB, et al.: Disorders related to sexuality and gender identity in the ICD-11: Revising the ICD-10 classification based on current scientific evidence, best clinical practices, and human rights considerations. World Psychiatry 2016;15:205-221.

60. Patton MQ: Qualitative Evaluation and Research Methods. Thousand Oaks, CA: Sage Publications, Inc., 1990, pp 169186.

61. Palinkas LA, Horwitz SM, Green CA, et al.: Purposeful sampling for qualitative data collection and analysis in mixed method implementation research. Adm Policy Ment Health 2015;42:533-544.

Address correspondence to: Athena D.F. Sherman, PhD Nell Hodgson Woodruff School of Nursing Emory University 1520 Clifton Road Atlanta, GA 30322

E-mail: adford4@emory.edu 